\title{
Percepção de sons de língua ESTRANGeira: QUESTÕES METODOLÓGICAS E O USO DOS APLICATIVOS PRAAT E TP
}

\section{Perception of nonnative sounds: methodological issues and the use of Praat and TP software}

\author{
Denise Cristina Kluge* \\ Andreia Schurt Rauber* \\ Anabela Rato** \\ Giane Rodrigues dos Santos ${ }^{* * * *}$
}

\begin{abstract}
RESUMO
O objetivo deste artigo é informar pesquisadores sobre alguns procedimentos metodológicos necessários para desenvolver testes de percepção e tarefas de treinamento perceptual. Após apresentar uma breve explicação sobre testes de discriminação e identificação, o artigo mostra como elaborar um teste perceptual em dois software livres diferentes: Praat e TP. Embora o Praat seja uma ferramenta excelente para elaborar experimentos de percepção, pode não ser um software amigável para pesquisadores que não se sintam confortáveis com a linguagem de programação e/ou com o inglês técnico utilizado no seu manual. Como uma alternativa, o TP foi criado para oferecer um software para testes/treinamento de percepção (áudio e audiovisual) que fornece feedback imediato e cuja configuração intuitiva segue a estrutura utilizada em aplicativos do Microsoft Windows.
\end{abstract}

Palavras-chave: testes de percepção; treinamento perceptual; software.

\footnotetext{
* Universidade Federal do Paraná.

"Appen Butler Hill.

"*tuniversidade do Minho.

".*tUniversidade Católica de Pelotas.
} 


\begin{abstract}
This paper aims at providing researchers with information about some methodological procedures to develop perception tests and perceptual training tasks. After presenting a brief explanation about identification and discrimination tests, the paper shows how to design a perceptual test in two different free applications: Praat and TP. Although Praat is an excellent tool to design perceptual experiments, it might not be a user-friendly software for researchers who are not comfortable with programming and/or the technical English language used in its manual. As an alternative, TP was designed to provide users with a software to test/train (audio and audiovisual) perception that gives immediate feedback and whose intuitive configuration follows the structure adopted in Microsoft Windows apps.
\end{abstract}

Keywords: perception tests; perceptual training; software.

\title{
1. Percepção de SONS de LÍNGUA ESTRANGeira
}

Pesquisas têm revelado que a percepção de segmentos e suprassegmentos de sons não nativos é um desafio para aprendizes de língua estrangeira/segunda (L2) (BEST; TYLER, 2007). Muitos fatores podem explicar essas dificuldades, como a influência do sistema fonológico da língua materna (L1), quantidade/qualidade de exposição à L2, idade do início da aprendizagem da L2, características individuais, entre outros. No entanto, um número considerável de estudos empíricos têm mostrado que falantes de L2 podem aprender a perceber contrastes não nativos de forma próxima a de nativos, uma vez que o mecanismos usado para aprender o sistema de sons da L1 pode ser utilizado para aprender os sons da L2 e "permanece intacto durante toda a vida ${ }^{1}$ " (FLEGE, 1995, p. 239, tradução nossa).

Um dos temas de pesquisas sobre a percepção da fala foca como o treinamento perceptual de segmentos e suprassegmentos de uma L2 podem modificar padrões da L1. Os resultados têm revelado que várias variáveis interagem para determinar até que ponto padrões perceptuais de uma determinada língua podem ser adaptados e modificados. De acordo com vários autores (JENKINS, 1979; STRANGE, 1992; BEDDOR; GOTTFRIED, 1995; LOGAN; PRUITT, 1995), há três tipos de variáveis em interação em qualquer procedimento experimental, identificado por Bohn (2000) como:

1 “...remain intact over the life span” (FLEGE, 1995, p. 239). 
variáveis relativas aos informantes, que definem o que um ouvinte traz para a tarefa de organizar perceptualmente contrastes não nativos (ex.: conhecimento da L1, experiência na L2, idade etc.); variáveis da tarefa, que definem os procedimentos experimentais utilizados para avaliar mudanças em padrões perceptuais (ex.: identificação ou discriminação, carga de memória em diferentes tarefas etc.); e variáveis quanto aos estímulos, que definem o que um ouvinte está tentando organizar perceptualmente (ex.: contrastes de consoantes ou vogais, pistas temporais ou espectrais etc.) (BOHN, 2000, p. 11) ${ }^{2}$.

Muitos estudos reportaram efeitos positivos do treinamento perceptual na modificação de padrões perceptuais (e.g., MCCLASKEY; PISONI; CARRELL, 1983; LIVELY et al., 1994) e na melhora da acurácia da produção (e.g., ROCHET, 1995; BRADLOW et al., 1997, 1999; YAMADA et al., 1999; WANG; JONGMAN; SERENO, 2003; LAMBACHER et al., 2005; NOBRE-OLIVEIRA, 2007; BRAWERMAN-ALBINI, 2012). De todos os estudos citados neste parágrafo, os únicos que investigaram os efeitos do treinamento perceptual tanto na percepção quanto na produção de sons de língua estrangeira (inglês) por brasileiros foram Nobre-Oliveira e Brawerman-Albini.

Nobre-Oliveira (2007) desenvolveu uma ferramenta para treinar a percepção das vogais do inglês /i, I, $\varepsilon$, æ, $v, \mathfrak{u}$ / por 29 brasileiros com nível intermediário de proficiência em língua inglesa. No seu estudo, os informantes recebiam um software desenvolvido pela própria pesquisadora para fazer o treinamento que continha dois conjuntos de tarefas perceptuais. $O$ treinamento era feito como tarefa de casa e poderia ser repetido quantas vezes o informante quisesse fazê-lo. Uma das limitações da ferramenta era a falta de feedback imediato aos informantes. Assim, a professora/ pesquisadora precisava corrigir as respostas manualmente e apenas então as correções eram enviadas aos alunos por e-mail. Apesar das limitações metodológicas, os resultados mostraram que a percepção dos informantes melhorou significativamente para os três pares de vogais após o treinamento e que a melhora na produção foi significativa apenas para o par de vogais /i//II/. Um teste de retenção revelou que a melhora se manteve um mês após o término do treinamento.

Brawerman-Albini (2012) investigou os efeitos de um treinamento perceptual do padrão acentual pré-proparoxítono da língua inglesa por 30

2 “...subject variables, which define what a listener brings to the task of perceptually organizing nonnative contrasts (e.g., L1 background, L2 experience, age, etc.); task variables, which define the experimental procedures used to assess changes in perceptual patterns (e.g., identification or discrimination, memory load in different tasks, etc.); and stimulus variables, which define what the listener is trying to organize perceptually (e.g., consonant or vowel contrasts, temporal or spectral cues, etc.)" (BOHN, 2000, p. 11). 
falantes brasileiros, divididos em Grupo Controle e Grupo Experimental. 0 treinamento perceptual, aplicado ao Grupo Experimental em cinco sessões, foi elaborado pela pesquisadora no aplicativo Microsoft Access e continha tarefas de identificação do acento com feedback imediato. Os resultados revelaram que o treinamento resultou em uma melhora significativa tanto da percepção como da produção das pré-proparoxítonas e que essa melhora ocorreu tanto nas palavras utilizadas no treinamento, como nas não utilizadas, evidenciando, assim, que a melhora na percepção obtida pelo treinamento perceptual é generalizada para novas palavras e retida pelo menos até dois meses após o término do treinamento.

Embora muitos estudos sobre a percepção de sons de L2 tenham sido conduzidos, o número de pesquisas envolvendo alunos brasileiros de línguas estrangeiras ou bilíngues ainda é reduzido (e.g., KOERICH, 2002; KLUGE, 2004, 2009; NOBRE-OLIVEIRA, 2007; BETTONI-TECHIO, 2008; GEWEHR-BORELLA, 2010; REIS, 2010; RESTREPO, 2011; BRAWERMAN-ALBINI, 2012). No Brasil, o interesse por pesquisa que investigue a percepção de sons tanto de $\mathrm{L} 1$ como de $\mathrm{L} 2$ tem aumentado consideravelmente. Um dos vários desafios encontrados pelos pesquisadores é a definição da metodologia necessária para testar os informantes de forma adequada e confiável. Ao seguir uma metodologia cuidadosa para a coleta de dados, espera-se, por exemplo, que os estímulos sonoros, ou seja, os sons a serem ouvidos, não sejam apresentados por meio de um toca CDs, sem fones de ouvido para cada um dos informantes. Portanto, se esse não é o procedimento ideal, quais ferramentas são normalmente usadas para testar informantes e quais os tipos de testes a serem desenvolvidos?

Neste artigo, discutiremos alguns desses aspectos. Na próxima seção, abordaremos diferentes tipos de apresentação de estímulos e também a importância do feedback sobre a performance dos informantes. Na Seção 3, mostraremos como elaborar um teste de percepção no software Praat (BOERSMA; WEENINK, 2013) e então apresentaremos uma nova ferramenta criada para auxiliar pesquisadores para elaborar experimentos de percepção mais intuitivamente: o TP (RAUBER; RATO; KLUGE; SANTOS, 2012). Finalmente, na Seção 4, reportaremos as considerações finais deste artigo.

\section{TESTES DE PERCEPÇÃo: CONCEITOS E SOFTWARE}

Nas últimas três décadas, a pesquisa sobre a percepção da fala tem utilizado diferentes procedimentos de testes/treinamento e vários aspectos têm sido considerados. Um deles é o tipo de teste a ser usado para apresentar os estímulos (LOGAN; PRUITT, 1995, p. 354-58). 
Um teste de discriminação, por exemplo, testa a diferenciação de dois ou mais estímulos e possui vários formatos: tarefas de categorização dos tipos $\mathrm{AX}, \mathrm{AXB}$ e $\mathrm{ABX}$, e teste de mudança categórica. Em um teste de discriminação categórica do tipo AX, um par de estímulos é apresentado e o informante deve indicar se ambos os estímulos são semelhantes/iguais ou diferentes. Por exemplo, ao se apresentar o par bet-bet (aposta-aposta) do inglês, espera-se que o informante perceba-o como contendo vogais semelhantes/iguais $([\varepsilon]-[\varepsilon])$; porém, ao se apresentar o par bet-bat (aposta-morcego), espera-se que o informante considere as vogais como diferentes, ou seja, pertencentes a duas categorias vocálicas distintas ([ع]-[æ]). Em um teste $\mathrm{AXB}, \mathrm{o}$ informante ouve uma sequência de três estímulos e decide se o segundo estímulo é semelhante ao primeiro ou ao terceiro. Por exemplo, o informante ouve a sequência bet-bat-bat ([ह]-[æ]-[æ]) e espera-se que perceba que a vogal do terceiro estímulo é semelhante à do segundo. Uma variação para esse teste é o formato $\mathrm{ABX}$, um teste de discriminação categórica com uma sequência de três estímulos, sendo que a posição do item diferente varia em cada sequência. $O$ informante deve decidir se o som diferente está na primeira, segunda ou terceira posição. Por exemplo, o informante ouve

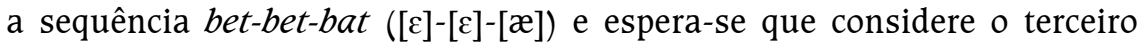
estímulo como contendo a vogal diferente dos demais. Há ainda o teste de mudança de categoria. Nesse teste, os informantes ouvem vários estímulos de uma categoria seguidos por estímulos de uma categoria diferente. Para exemplificar, o informante ouve uma sequência de estímulos "ba"-"ba"-"ba" e, quando é apresentado um estímulo "da", o informante clica em um botão de resposta, assinalando que houve mudança de categoria ([b]-[d]).

Em relação a testes de identificação, um único estímulo é apresentado e o informante deve identificá-lo, geralmente escolhendo entre várias opções de respostas que podem ser apresentadas em uma folha de papel ou na tela de um computador. Por exemplo, um informante ouve a palavra inglesa bet e identifica qual das opções de resposta disponíveis "[er]”, "[ع]" ou "[æ]" é a que mais se assemelha à vogal ouvida.

Além do tipo de teste, outro aspecto a ser considerado ao se elaborar um teste de percepção é o tipo de estímulo. O pesquisador pode optar por estímulos naturais, gravados por um falante humano, ou estímulos sintetizados, normalmente gerados por uma máquina e cujas pistas (e.g., duração e/ou qualidade espectral) são manipuladas de acordo com o objetivo da pesquisa.

Um terceiro aspecto a ser controlado é o número de locutores que gravarão os estímulos. Para testar a discriminação ou identificação, aconselha-se que sejam gravados estímulos por três locutores de gêneros diferentes para assegurar que, independentemente da qualidade da voz ou- 
vida, o informante seja capaz de discriminar entre categorias ou identificar uma determinada categoria.

Em relação ao treinamento, experimentos de treinamento perceptual de L2 costumam utilizar as mesmas tarefas adotadas na área mais ampla de pesquisa sobre percepção da fala, nomeadamente tarefas de identificação e discriminação; porém, há algumas variações. Uma das diferenças entre uma tarefa de teste perceptual e de treinamento perceptual é que esta costuma fornecer feedback quanto à performance dos informantes após a apresentação de cada estímulo ou de um grupo de estímulos. O feedback é considerado importante porque torna o treinamento mais eficiente, indicando se os informantes perceberam as diferenças categóricas ou suprassegmentais adequadamente ou não.

o feedback pode ser fornecido após um período de tempo curto ou longo. No primeiro caso, os informantes recebem informação sobre a sua resposta imediatamente após ouvirem e responderem à cada estímulo, o que é chamado de feedback por estímulo (stimulus-by-stimulus feedback). No segundo caso, a informação fornecida compreende um número maior de estímulos e é chamada de feedback cumulativo (cumulative feedback). o feedback cumulativo pode ser do tipo em que a resposta é fornecida aos informantes após a apresentação de um bloco de estímulos ou após o término de cada sessão de treinamento.

O treinamento perceptual também varia de acordo com a duração (curta ou longa) e tipo (apenas auditivo, apenas visual ou audiovisual). Dependendo do segmento a ser testado, pistas visuais podem ser úteis para aumentar a conscientização (awareness) do informante sobre as diferenças entre categorias. Kluge (2009), por exemplo, aplicou testes nas condições apenas áudio, apenas vídeo e audiovisual para testar a percepção das nasais [m] e [n] do inglês em posição final de palavras por brasileiros. Nas últimas duas condições, os informantes visualizavam os movimentos da boca de um americano articulando as consoantes-alvo em palavras como "Tim" ou "Tin". Os resultados revelaram que a condição de treinamento audiovisual favoreceu significativamente a percepção dos estímulos-alvo, enquanto que a condição apenas áudio não favoreceu a percepção desses segmentos.

Como observamos, para treinar falantes a perceberem categorias fonéticas não nativas podemos empregar vários tipos de tarefas de treinamento perceptual. Com o avanço da tecnologia, pesquisadores têm desenvolvido diversos aplicativos de treinamento perceptual, sendo frequentes os elaborados no software livre Praat ou em aplicativos do Microsoft Windows. Se o pesquisador tiver acesso a um laboratório, o uso de software para treinamento perceptual permite que se teste um grande grupo de informantes ou alunos simultaneamente. Enquanto esse tipo de software facilita o treinamento/ 
teste e reduz o tempo de coleta e análise de dados, já que as respostas são armazenadas automaticamente em tabelas, um desafio é a elaboração dos testes ou das tarefas, que não costuma ser um processo simples e intuitivo. Para ilustrarmos esse processo, na próxima seção compararemos o design de um teste de identificação em dois software livres: Praat e TP.

\section{Elaborando testes de Percepção nos softwares livres PraAt e TP}

O principal objetivo desta seção é mostrar os desafios com os quais um pesquisador pode se deparar ao elaborar um teste de percepção. Após tomar todas as decisões sobre a metodologia ideal para o seu experimento (e.g., foco de estudo, número de estímulos, número de locutores que gravarão os estímulos, contextos fonológicos nos quais os segmentos-alvo serão inseridos, tipo de teste, natureza dos estímulos, número de vezes que cada estímulo poderá ser repetido pelo informante), a elaboração do teste pode ser um tanto difícil principalmente para linguistas que não estão familiarizados com a linguagem de programação. Um software livre amplamente utilizado para se aplicar testes de percepção é o Praat. A Figura 1 mostra a tela de um teste de identificação elaborado no Praat, cujo objetivo é testar a percepção das vogais do português.

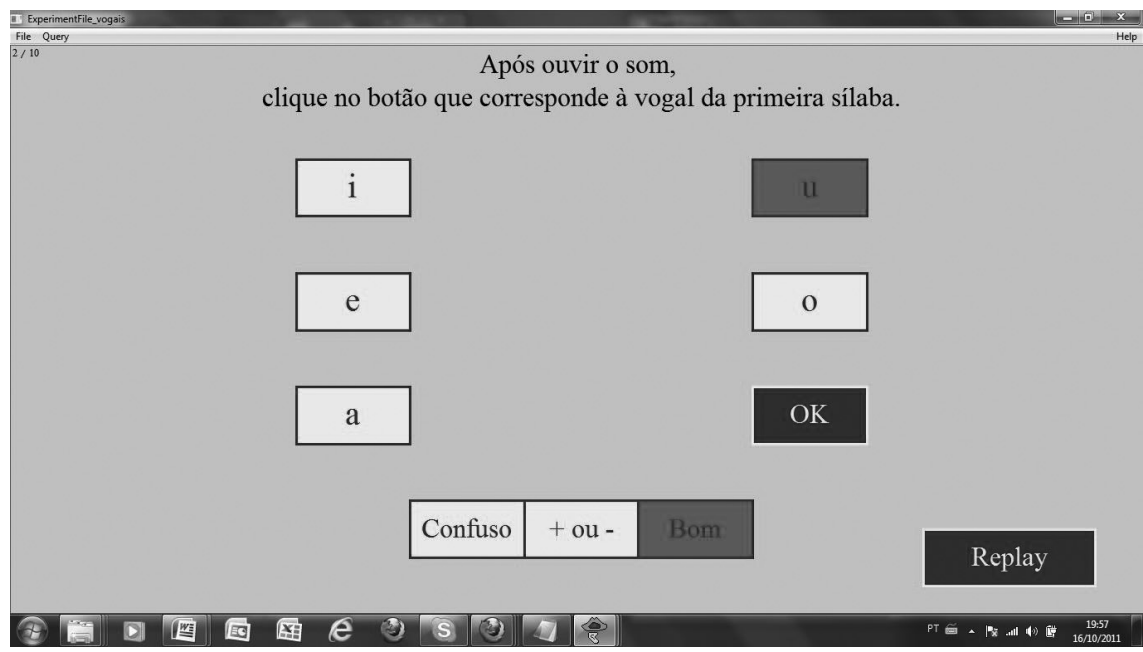

FIGURA 1 - Tela de um teste de identificação elaborado no Praat para testar a percepção de vogais do português 
Para rodar o teste de percepção apresentado na Figura 1, as linhas de programação abaixo devem ser digitadas em um arquivo de experimento (experiment file) do Praat. Um arquivo de experimento pode ser aberto de duas formas: como um script, que permitirá modificações nos comandos através do Menu "Praat", opção "Open Praat script...", e como o próprio experimento, que deve ser aberto através do Menu "Open", opção "Read from file...". Para editar as linhas a seguir, o arquivo que as contêm deve ser aberto como um script.

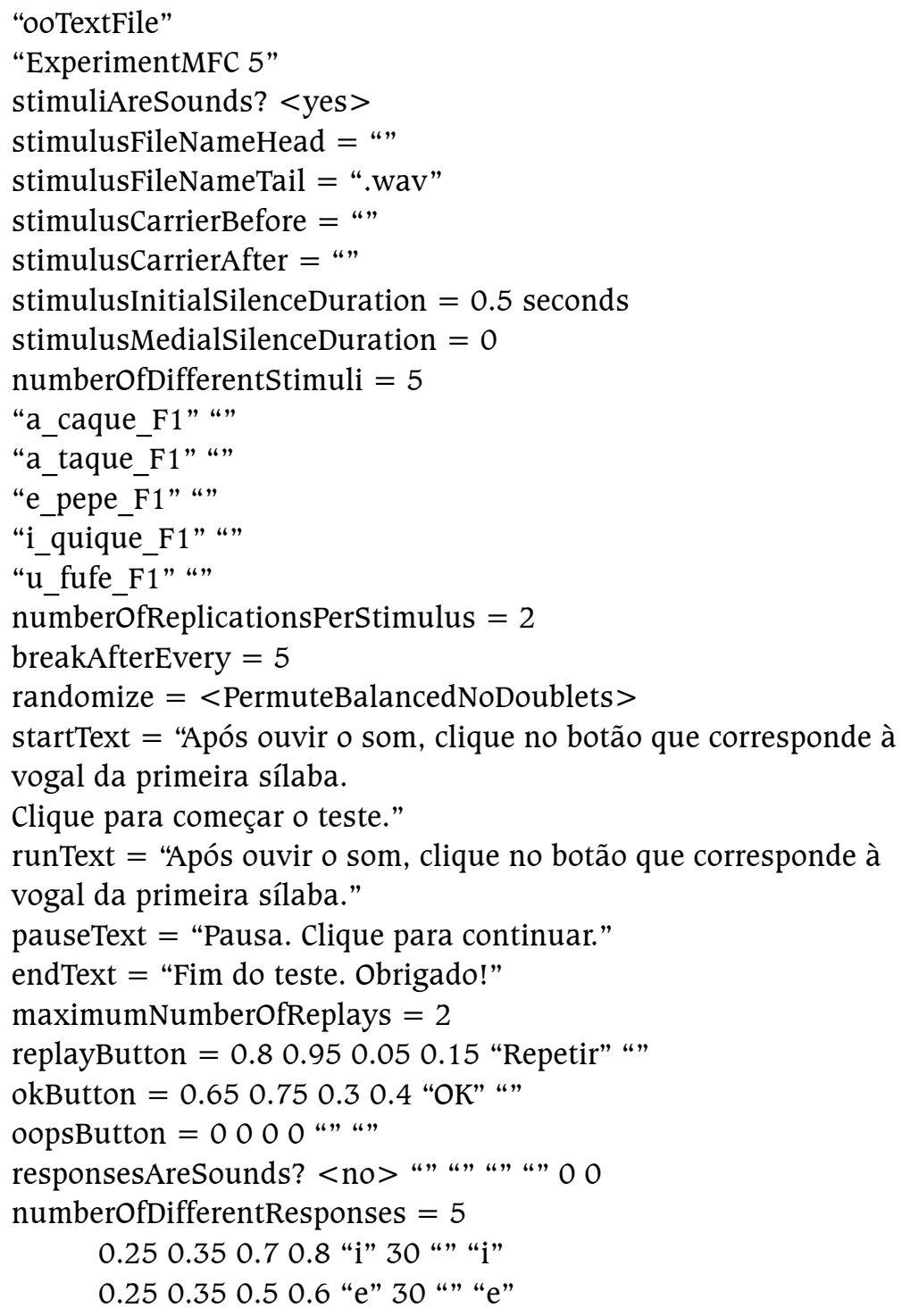


0.250 .350 .30 .4 “a” 30 “" "a”

0.650 .750 .70 .8 “u” 30 " " "u"

0.650 .750 .50 .6 "o" 30 “" "o"

numberOfGoodnessCategories $=3$

0.350 .450 .100 .20 "Confuso"

0.450 .550 .100 .20 "+ ou -"

0.550 .650 .100 .20 "Bom”

Como podemos observar, sem a leitura cuidadosa do manual do Praat, um pesquisador que não esteja familiarizado com os comandos de programação terá dificuldades para entender como adaptar o script para os seus propósitos. De forma sucinta, as duas primeiras linhas indicam que se trata de um experimento de percepção e não devem ser alteradas. As próximas sete linhas definem 1) que os estímulos a serem ouvidos serão sonoros, 2) qual a extensão do arquivo ("Tail") a ser usada, e 3) se haverá alguma frase-veículo tanto antes como após o estímulo-alvo. Duas aspas seguidas (“") indicam que não há informação para um determinado comando, ou seja, para especificar que um dos comandos não será utilizado, não se deve simplemente apagar a linha equivalente ao comando, mas apenas informar com duas aspas duplas que esse comando não é necessário. Na décima linha, o comando "numberofDifferentstimuli" indica o número de arquivos com estímulos sonoros a serem apresentados e esse número deve corresponder aos nomes dos arquivos de som, que são listados logo abaixo dessa linha de comando (linhas 11 a 15). O comando na linha 16 (numberofReplicationsPerStimulus $=2$ ) define o número de vezes que o estímulo será apresentado aos informantes, nesse exemplo, duas vezes. Na linha 17 , o comando "breakAfterEvery = 5" permite que uma pausa seja inserida após um dado número de estímulos ser apresentado, nesse caso, após cinco estímulos. 0 comando "randomize" na linha 18 é de grande ajuda aos pesquisadores, pois aleatoriza todos os estímulos automaticamente, o que é um procedimento metodológico importante para se evitar efeitos de ordenamento de estímulos, isto é, efeitos que podem comprometer a pesquisa porque os dados são sempre apresentados em uma determinada sequência. Os comandos nas linhas 19 a 25 definem os textos a serem exibidos: 1) na tela de apresentação do teste, 2) nas telas do teste, 3) na tela de pausa e 4) na tela de encerramento do teste. $O$ comando na linha 26 (maximumNumberofReplays $=3$ ) define o número de vezes que os informantes poderão repetir um estímulo ouvido. Nesse exemplo, o número máximo de repetições está limitado a duas e após a segunda repetição o botão "Repetir" desaparece da tela do teste. Os comandos nas linhas 27 a 39 definem a posição dos botões "Repetir", "OK", e "Oops". O primeiro, conforme já explicado, permite que os informantes repitam um estímulo ou uma sequência de estímulos. O segundo, "OK", 
deve ser pressionado após um botão de resposta ser escolhido para apenas então o próximo estímulo ser apresentado. o terceiro, "Oops", permite que os informantes repitam e troquem a resposta do estímulo anterior ao atual, o que é útil quando se percebe que se cometeu um erro na escolha da resposta do estímulo anterior. Caso o pesquisador não queira usar nenhum desses botões, ele pode optar por desabilitá-los, utilizando zero nas coordenadas e deletando as informações que estão entre aspas (nesse exemplo, o botão Oops está desabilitado, por isso a linha contém zeros e nenhuma informação entre aspas: oopsButton = 0000 "” "”). Como já dito, no caso da preferência por desabilitar um botão, não podemos simplesmente apagar a linha de comando ou inserir o símbolo cardinal (\#) no início da linha, como se faz nos demais scripts do Praat para se desabilitar um comando, pois o script não funcionará sem uma das linhas. O comando na linha 30 (responsesAreSounds?) deve ser deixado em branco se o objetivo do teste for o de oferecer aos informantes a opção de escolher um botão ou etiqueta como resposta, isto é, se o botão de resposta não tocará nenhum som quando clicado. 0 comando na linha 31 (numberofDifferentResponses $=5$ ) define o número de botões de resposta a serem exibidos na tela do computador; nesse exemplo, há cinco botões. As coordenadas de localização de cada botão são definidas nos comandos das linhas 32 a 36. Nesse exemplo, a informação na linha 32 (0.25 0.350 .70 .8 "i" 30 “" "i”) indica que uma caixa retangular será desenhada do ponto 0.25 da coordenada da esquerda até o 0.35 da direita e do ponto 0.7 da coordenada inferior ao 0.8 da superior. A letra "i" será a etiqueta a ser exibida no botão de resposta e terá o tamanho de fonte "30". As aspas seguidas, sem informação entre elas, indicam que nenhuma tecla do teclado será utilizada para escolher a resposta certa, isto é, os informantes terão que clicar no botão de resposta com o mouse. Finalmente, o último "i" nessa linha indica que ao clicar no botão cuja etiqueta é "i", a resposta a ser registrada na tabela de respostas será o símbolo "i". O comando na linha 37 (numberofGoodnessCategories) define o número de itens de uma Escala de Likert utilizada para julgar a qualidade do estímulo de uma dada categoria fonética. Nesse exemplo, há três categorias: a) se o informante considera que o estímulo é um bom exemplar de uma determinada categoria; b) se é um exemplar mais ou menos bom; ou c) se é um exemplar ruim.

Após os comandos serem programados adequadamente, o script deve ser salvo e então aberto como um arquivo de experimento (via Menu open, Read from file...). Quando um arquivo de experimento está aberto na caixa "Praat object", o pesquisador deve clicar no botão "Run". Após rodar o experimento, o pesquisador deve clicar no botão "Extract results", e na sequência "Collect to table", e então salvar o arquivo criado clicando no Menu "Save", opção "Save as tab-separated file...". o arquivo com as respostas exibirá o nome do arquivo correspondente a cada estímulo, a opção 
de resposta escolhida pelo informante e o tempo que o informante levou para clicar em uma opção após ouvir cada estímulo (tempo de reação). Após esses procedimentos, os resultados estarão prontos para serem computados.

Embora o Praat seja uma excelente ferramenta para desenvolver testes de percepção, não é o software ideal a ser usado para treinamento perceptual, pois os arquivos de experimento não permitem que se forneça feedback imediato aos informantes. Devido a essa limitação e também considerando-se os desafios encontrados por pesquisadores que não estão familiarizados com a linguagem de programação e/ou com o inglês e linguagem técnica utilizada no manual do Praat, desenvolvemos o software $\mathrm{TP}^{3}$, cuja sigla significa Testes/Treinamentos de Percepção.

Dentre as várias funções do TP, as principais características são que o software a) permite a aplicação de dois tipos de testes de percepção: discriminação e identificação; b) fornece feedback após cada estímulo e também feedback cumulativo; c) mede o tempo de reação para cada resposta; d) permite que sejam aplicados testes com estímulos sonoros e audiovisuais; e) aleatoriza os estímulos, criando uma nova ordem de apresentação toda vez que o teste é reiniciado; f) salva as respostas em arquivos do Excel, já apontando quais são as respostas corretas e incorretas; e g) possui a opção de enviar um experimento completo a outros computadores, facilitando, assim, a instalação de testes em laboratórios com várias máquinas.

Uma das preocupações ao elaborarmos o TP foi a de garantir a facilidade de manuseio por parte de pesquisadores e/ou professores de línguas. O software pode ser rodado nos modos "Professor" e "Aluno". A configuração e edição podem ser feitas apenas pelo pesquisador, enquanto que o usuário (aluno/informante) está limitado a fazer os testes, ou seja, o seu acesso à configuração e respostas dos testes é bloqueado por uma senha.

Como exibido na Figura 2, há quatro menus principais no aplicativo: Parâmetros, Aplicação, Sobre e Sair. Descreveremos as funções apenas do Menu Parâmetros.

No Menu Parâmetros, no botão Configuração dos Testes, o pesquisador pode:

1. configurar/selecionar o idioma no qual o teste será aplicado;

2. defininir se o teste a ser aplicado será rodado em modo teste (sem feedback) ou treinamento (com feedback);

3. ativar a opção de Escala de Likert (goodness-offfit) limitada a nove pontos;

4. ativar o botão "Oops" (no caso de o informante perceber que cometeu um erro ao selecionar a resposta ao item anterior, esse botão permite apagar a resposta anterior e ouvir esse estímulo novamente); 


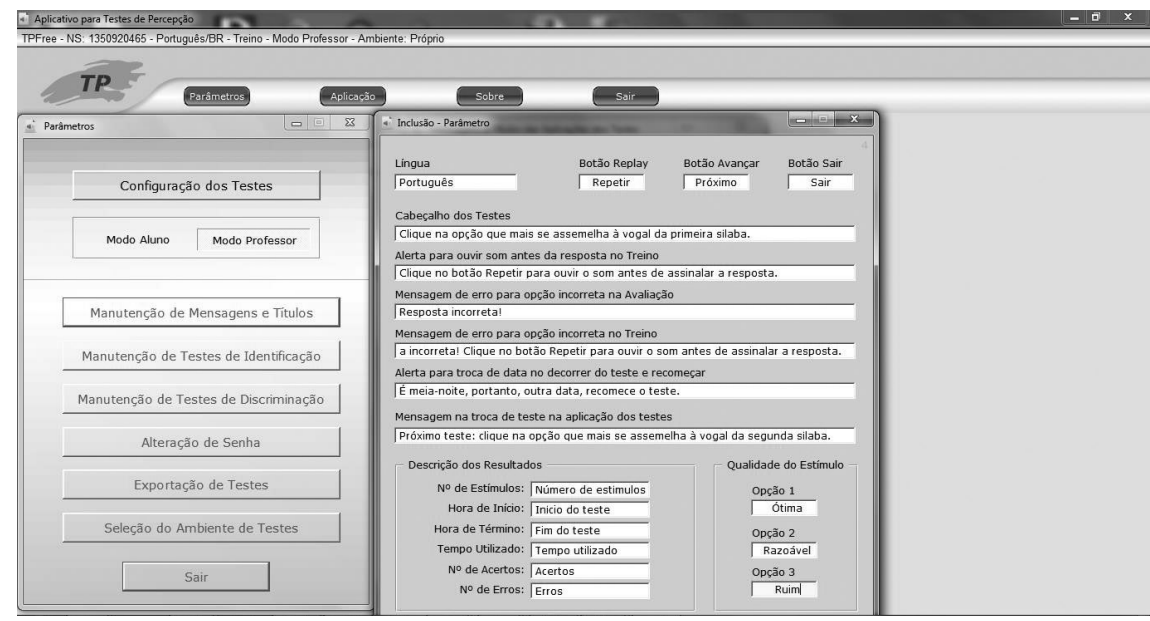

FIGURA 2 - Janelas do TP que mostram os menus principais e a configuração das mensagens e botões em qualquer idioma, neste exemplo, no português brasileiro

5. definir se o teste a ser aplicado será apenas com estímulos sonoros ou se será com estímulos audiovisuais, ou ainda se os botões de resposta serão figuras e não texto.

Ainda no Menu Parâmetros, é possível configurar o teste no Modo Professor (com acesso ao menu de configuração) ou no Modo Aluno (sem acesso a respostas ou configuração). É nesse menu que está a opção para configurar as mensagens informativas em cada tela do teste e também o texto a ser apresentado nos botões de comando.

A Figura 3 ilustra as opções de configuração acessadas pelo botão "Manutenção de Testes de Identificação".

Como podemos visualizar na parte superior da Figura 3, cada teste tem um número, um título e um botão que permite ativá-lo ou desativá-lo. Os estímulos (arquivos .wav) são carregados ao clicar-se no símbolo "+", que pode ser encontrado no canto inferior esquerdo da figura. Ao clicar nesse botão, uma janela de inclusão de estímulos permite que o usuário localize arquivos de som em seu computador e defina qual o número do botão que corresponde à resposta correta para aquele arquivo. O texto dos botões é definido na área "Opções de resposta". O número de botões de respostas é limitado a 28. Embora não possamos visualizar na Figura 4, é nessa tela que definimos o número de vezes que o mesmo estímulo será tocado no teste, o número de repetições por estímulo permitidas ao se clicar no botão "Repetir", e o número de estímulos que são tocados até que haja uma pausa no teste. As mesmas opções para configurar testes de identificação estão disponíveis para configurar testes de discriminação. 


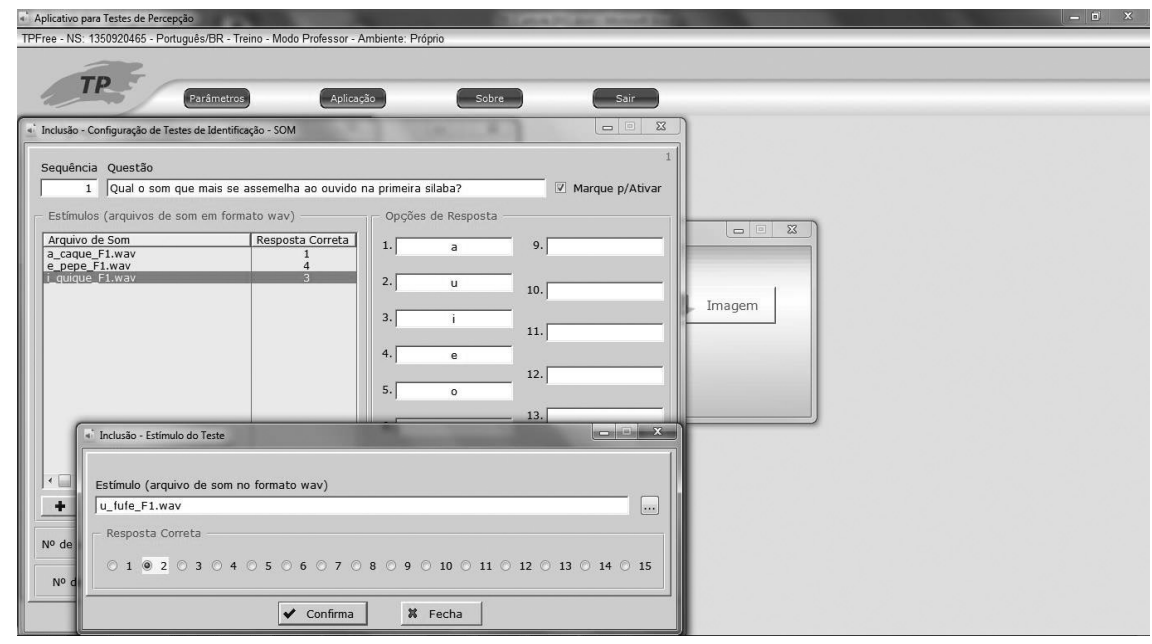

FIGURA 3 - Janelas do TP que ilustram a configuração dos botões de resposta e a forma como os arquivos de som são carregados e a resposta correta definida

O resultado da configuração do teste de identificação descrito acima é apresentado ao informante/aluno conforme ilustra a Figura 4. Nesse exemplo, o estímulo tocado continha uma palavra cuja vogal da primeira sílaba era $[u]$, porém, o informante clicou em um botão de resposta incorreto. Como, no exemplo, o teste está configurado no Modo Treinamento, a resposta correta é assinalada assim que o informante clica no botão incorreto e uma mensagem de erro orienta-o a ouvir o estímulo novamente para apenas então clicar no botão correto. No caso de erro, o software não permite que o

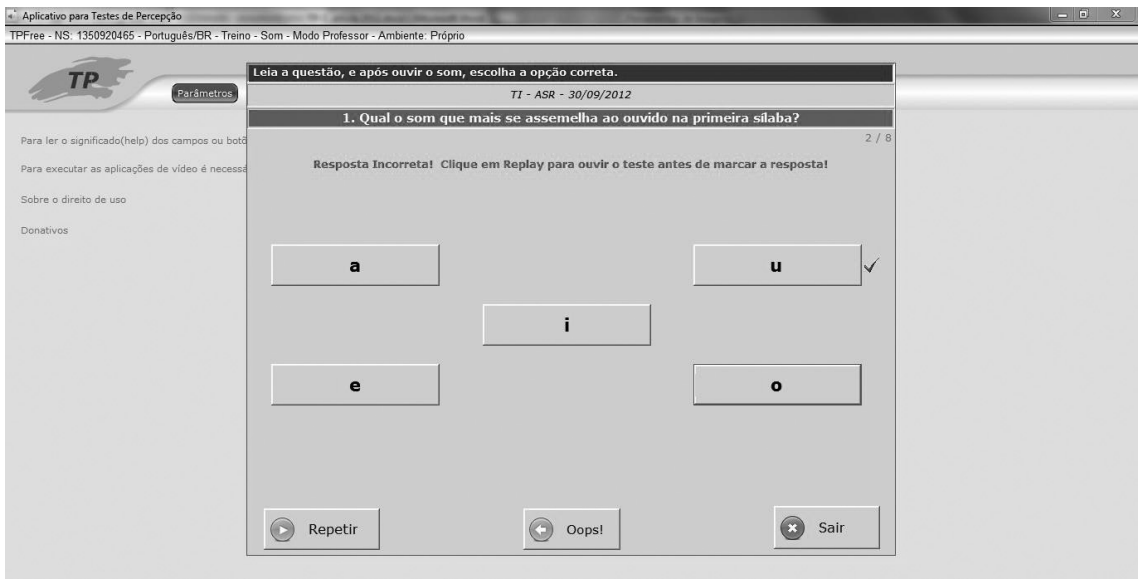

FIGURA 4 - Tela de um teste de identificação do TP no modo treinamento (feedback imediato) 
informante avance sem ouvir o estímulo outra vez. O número de repetições permitido é pré-definido pelo pesquisador quando configura o teste. Após terminar o teste, uma janela com o número de acertos, erros e o tempo de duração do teste é apresentada ao informante.

Os mesmos procedimentos de configuração são adotados para elaborar testes áudio/audiovisuais e testes cujos botões de respostas são imagens. As Figuras 5 e 6 ilustram as telas de cada teste, respectivamente.

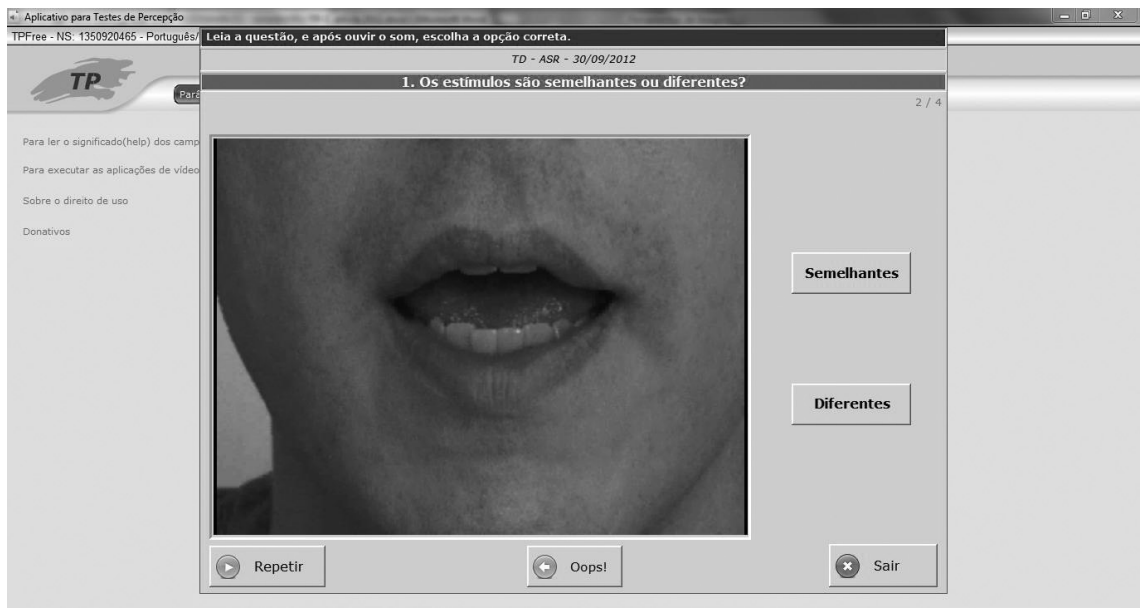

FIGURA 5 - Tela do TP ilustrando um teste de discriminação com estímulos audiovisuais

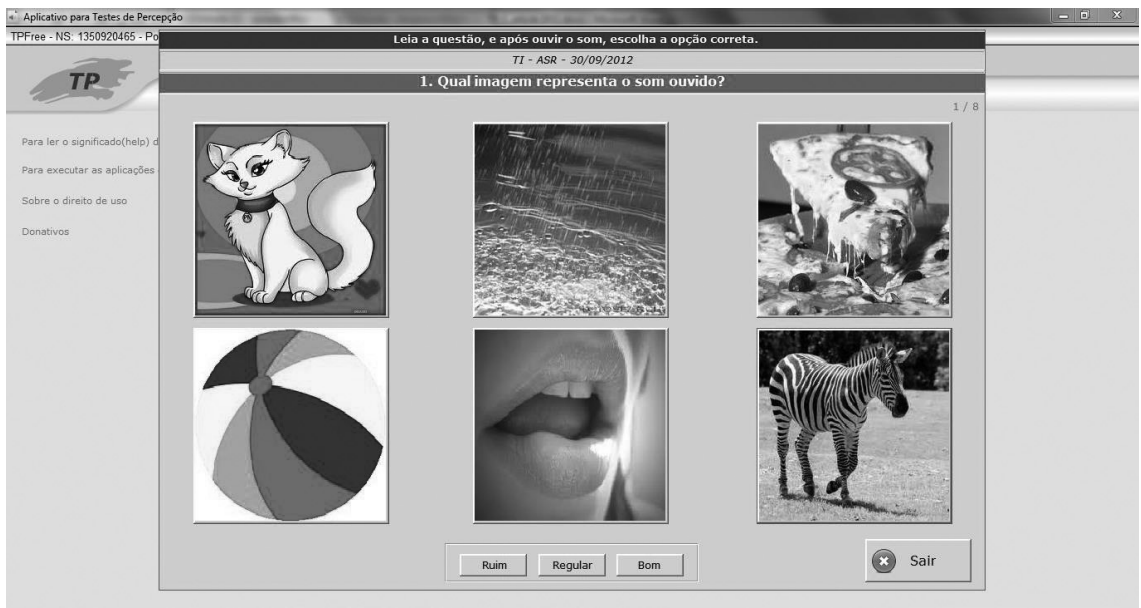

FIGURA 6 - Tela do TP ilustrando um teste de identificação com figuras como botões de resposta 
Ao concluir cada teste, um arquivo é automaticamente criado no Excel (Figura 7) com informações sobre os acertos e o tempo de reação de cada estímulo, bem como sobre a avaliação da qualidade do segmento ouvido, caso a Escala de Likert (goodness-of-fit) tenha sido ativada.

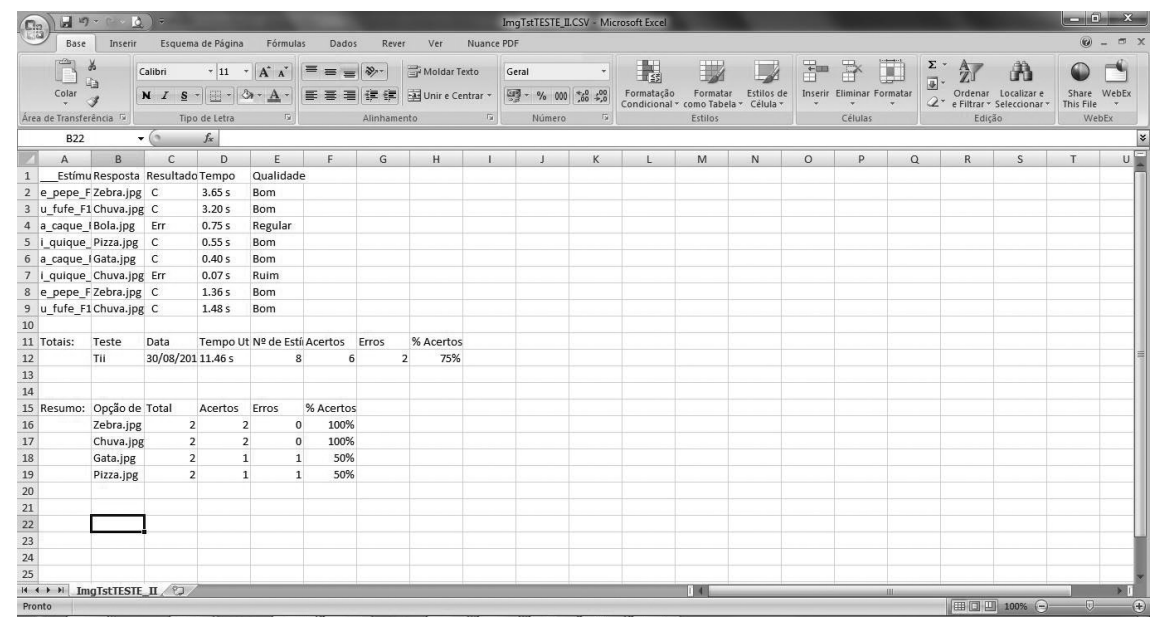

FIGURA 7 - Arquivo gerado no Excel com informações sobre as respostas dos informantes e tempo de reação para a seleção de cada estímulo

Ainda no Menu Parâmetros, é possível exportar um teste, criando uma pasta .zip com os arquivos de configuração do teste e os arquivos de som. Isso permite que o teste configurado em um computador seja instalado em outros computadores de um laboratório ou enviado a outros pesquisadores/alunos por e-mail. Caso o pesquisador queira enviar o teste na sua forma final a informantes/alunos, basta clicar na opção TP Direct, que exportará os experimentos ativados/selecionados sem permitir qualquer edição. Assim, essas duas opções de exportação permitem que pesquisadores enviem experimentos tanto a outros pesquisadores que precisam ter acesso às configurações como aos informantes.

Nesta seção comparamos os procedimentos utilizados para configurar testes/treinamentos de percepção em dois software livres: Praat e TP. A próxima seção trará as considerações finais deste artigo.

\section{Considerações FinAis}

Considerando-se o interesse crescente por pesquisas sobre percepção da fala (L1 e L2), neste artigo abordamos alguns procedimentos metodo- 
lógicos importantes para elaborarmos tanto testes de percepção como tarefas de treinamento perceptual. Preocupadas com as dificuldades encontradas por alunos de graduação e pós-graduação das áreas de Linguística, Linguística Aplicada, Psicolinguística e Fonoaudiologia, criamos um software livre que simplifica o design de testes/treinamentos de percepção: o TP. Reconhecemos a indiscutível relevância do software Praat, que inspirou o TP, mas objetivamos oferecer a pesquisadores/professores uma ferramenta mais amigável, especialmente pensada para aqueles que não estão familiarizados com a linguagem de programação e não se sentem confortáveis com a linguagem técnica do manual do Praat. Embora haja algumas diferenças, ambos os aplicativos são extremamente úteis e confiáveis para a elaboração de testes de percepção; porém, o TP ainda permite o design de tarefas de percepção com feedback imediato e a elaboração de testes/tarefas contendo estímulos audiovisuais.

Após vários anos de pesquisa na área de percepção da fala, pretendemos motivar e facilitar o trabalho de pesquisadores e professores de idiomas ao conduzirem experimentos nessa área, acumulando, assim, mais conhecimento sobre a percepção de propriedades acústicas de sons nativos e não nativos.

\section{REFERÊNCIAS}

BEDDOR, Patrice; GOTTFRIED, Terry. Methodological issues in cross-language speech perception research with adults. In: STRANGE, W. (Ed.). Speech perception and linguistic experience: issues in cross-language research. Timonium, MD: York Press, 1995. p. 207-232.

BEST, Catherine; TYLER, Michael. Nonnative and second-language speech perception: commonalities and complementarities. In: BOHN, O.; MUNRO, M. (Ed.). Language experience in second language speech learning: in honor of James Emil Flege. Amsterdam/Philadelphia: John Benjamins, 2007. p. 13-34.

BETTONI-TECHIO, Melissa. Perceptual training and word-initial/s/-clusters in Brazilian Portuguese/English interphonology. Tese (Doutorado em Letras/Inglês) - Programa de Pós-Graduação em Inglês, Universidade Federal de Santa Catarina, Florianópolis, 2008.

BOERSMA, Paul; WEENINK, David. Praat: doing phonetics by computer, v. 5.3.56. Disponível em: <www.praat.org>. Acesso em: 15/9/2013.

BOHN, Ocke-Schwen. Linguistic relativity in speech perception: an overview of the influence of language experience on the perception of speech sounds from infancy to adulthood. In: NIEMEIER, S.; DIRVEN, R. (Ed.). Evidence for linguistic relativity, v. 98. Amsterdam, Philadelphia: John Benjamins, 2000. p. 1-28.

BRADLOW, Ann; PISONI, David B.; YAMADA, Reiko A.; TOHKURA, Yohichi. Training Japanese listeners to identify English /r/ and /l/: IV. Some effects of perceptual learning on speech production. Journal of the Acoustical Society of America, v. 101, p. 2299-2310, 1997.

BRADLOW, Ann; YAMADA, Reiko; PISONI, David; TOHKURA, Yohichi. Training Japanese listeners to identify English /r/ and /l/: Long-term retention of learning in perception and production. 
Perception \& Psychophysics, v. 61, p. 977-985, 1999.

BRAWERMAN-ALBINI, Andressa. Os efeitos de um treinamento de percepção na aquisição do padrão acentual pré-proparoxítono da língua inglesa por estudantes brasileiros. Tese (Doutorado em Letras) - Programa de Pós-Graduação em Letras, Universidade Federal do Paraná, Curitiba, 2012.

FLEGE, James Emil. Second language speech learning: theory, findings and problems. In: STRANGE, W. (Ed). Speech perception and linguistic experience: issues in cross-language research. Timonium, MD: York Press, 1995. p. 233-277.

GEWEHR-BORELLA, Sabrina. A influência da fala bilíngue hunsrückisch-português brasileiro na escrita de crianças brasileiras em séries iniciais. Dissertação (Mestrado em Letras) - Programa de Pós-Graduação em Letras, Universidade Católica de Pelotas, Pelotas, 2010.

JENKINS, James. Four points to remember: a tetrahedral model of memory experiments. In: CERMAK, L. S.; CRAIK, F. I. M. (Ed.). Levels of processing in human memory. Hillsdale, NJ: Erlbaum, 1979. p. 429-446.

KLUGE, Denise Cristina. Perception and production of English syllable-final nasals by Brazilian learners. Dissertação (Mestrado em Letras/Inglês e Literatura Correspondente) - Programa de Pós-Graduação em Inglês, Universidade Federal de Santa Catarina, Florianópolis, 2004.

KLUGE, Denise Cristina. Brazilian EFL learners' identification of word-final /m-n/: native/ nonnative realizations and effect of visual cues. Tese (Doutorado em Letras/Inglês) - Programa de Pós-Graduação em Inglês, Universidade Federal de Santa Catarina, Florianópolis, 2009.

KOERICH, Rosana Denise Perception and production of word-final vowel epenthesis by Brazilian EFL students. Tese (Doutorado em Letras/Inglês) - Programa de Pós-Graduação em Inglês, Universidade Federal de Santa Catarina, Florianópolis, 2002.

LAMBACHER, Stephen; MARTENS, William; KAKEHI, Kazuhiko; MARASINGHE, Chandrajith; MOLHOLT, Garry. The effects of identification training on the identification and production of American English vowels by native speakers of Japanese. Applied Psycholinguistics, v. 26, p. 227-247, 2005.

LIVELY, Scott E.; PISONI, David; YAMADA, Reiko; TOHKURA, Yohichi.; YAMADA, T. Training Japanese listeners to identify English /r/ and /1/: III. Long-term retention of new phonetic categories. Journal of the Acoustical Society of America, v. 96, p. 2076-2087, 1994.

LOGAN, James; PRUITT, Jessica. Methodological issues in training listeners to perceive nonnative phonemes. In: STRANGE, W. (Ed.). Speech perception and linguistic experience: issues in cross-language research. Timonium, MD: York Press, 1995. p. 351-378.

MCCLASKEY, C. L.; PISONI, David. B.; CARRELL, T. D. Transfer of training of a new linguistic contrast in voicing. Perception and Psychophysics, v. 34, p. 323-330, 1983.

NOBRE-OLIVEIRA, Denise. The effect of perceptual training on the learning of English vowels by Brazilian Portuguese speakers. Tese (Doutorado em Letras/Inglês) - Programa de Pós-Graduação em Inglês, Universidade Federal de Santa Catarina, Florianópolis, 2007.

RAUBER, Andréia S.; RATO, Anabela; KLUGE, Denise C.; SANTOS, Giane R. dos. TP: Testes/ Treinamentos de Percepção [software]. Disponível em: <http://www.worken.com.br/tp_regfree. php>. Acessado em: 25/8/2012.

RAUBER, Andréia S.; RATO, Anabela; KLUGE, Denise C.; SANTOS, Giane R. dos. TP 3.1 Software: A tool for designing audio, visual, and audiovisual perceptual training tasks and perception tests. Proceedings of Interspeech 2013, p. 2095-2098, 2013.

REIS, Mara Silvia. The perception and production of the English voiceless interdental fricative by speakers of European French and Brazilian Portuguese. Tese (Doutorado em Letras/Inglês) - Programa de Pós-Graduação em Inglês, Universidade Federal de Santa Catarina, Florianópolis, 2010. 
RESTREPO, Júlia. Percepção e produção de aprendizes brasileiros de francês: o caso das vogais médias anteriores arredondadas. Dissertação (Mestrado em Linguística) - Programa de Pós-Graduação em Linguística, Universidade Federal de Santa Catarina, Florianópolis, 2011.

ROCHET, B. Perception and production of second-language speech sounds by adults. In: STRANGE, W. (Ed.). Speech perception and linguistic experience: issues in cross language research. Timonium, MD: York Press, 1995. p. 379-410.

STRANGE, Winifred. Learning non-native phoneme contrasts: interactions among subject, stimulus, and task variables. In: TOKHKURA, Y.; VATIKIOTIS-BATESON, E.; SAGISAKA, Y. (Ed.). Speech perception, production and linguistic structure. Tokyo: Om, 1992. p. 197-219.

WANG, Yue; JONGMAN, Allard; SERENO, Joan. Acoustic and perceptual evaluation of Mandarin tone productions before and after training. Journal of the Acoustical Society of America, v. 113, p. 1033-1043, 2003.

YAMADA, Reiko; TOHKURA, Yohichi; BRADLOW, Ann; PISONI, David. Does training in speech perception modify speech production? In: Proceedings of the International Congress of Phonetic Sciences, San Francisco, 1999. p. 117-120.

Submetido em: 09/11/2013

Aceito em: 27/01/2014 